\title{
1 Position of the Problem
}

After presentation, in the previous part of an input-output table in the context of illbehaved inverse problem solution, let us talk about the next national accounts table, known as a Social Accounting Matrix (SAM) (e.g., Stone, 1970; Graham, 1985; Scandizzoa \& Ferrareseb, 2015). From a macroeconomic point of view, this table is a generalization of an input-output table. While input-output shows primary distribution of income without saying anything about its secondary distribution, the SAM table fills in this missing information and, at the same time, displays a complete flow of products and values in a general equilibrium framework. In the coming section, the ill-behaved inverse problem will be particularly addressed since SAM table building, more than for input-output tables, requires more information to be gathered from different sources but involving, consequently, higher risk of statistical inconsistencies when this information is aggregated. In this chapter, we present the general aspects of a SAM by introducing the parameter or multiplier estimation approach, and we then discuss the limits of classical econometric methods.

For the last two decades, Kullback-Leibler minimum entropy (KLME) formalism has encountered relative success in the social sciences, particularly when a solution was required for inverse problems (e.g., Robinson et al., 2001; Bwanakare, 2013). The objective of the present document is to extend the KLME approach to the non-ergodic Tsallis entropy system, represented in the present study by an initially non-balanced quadratic social accounting matrix (SAM), known to display Walrasian general equilibrium features.

The described economic system is then defined by different interactive subsystems, each represented by respective actors and characterized by optimizing behaviour. Households, which tend to maximize a certain utility function, remain the owner of factors of production and are the final consumer of produced commodities while firms maximize profits by optimal renting of these factors from households for the production of goods and services. In this model, government has the passive role of collecting taxes and disbursing tax revenue. Furthermore, the economy analysed is small and open and a 'price taker' from the rest of the world. The above optimal behaviour inside subsystems leads to general market equilibrium in all respective sectorial markets.

A SAM table is a statistical tool to summarize all the above economic transactions by registering, in their respective rows and columns income and expenses in accordance with the double-entry book-keeping principle. However, due to different and sometimes contradictory sources of collected statistical information, the SAM is not balanced, i.e., respective column or row totals are not matching. Such statistical data may display, as partially coming from statistical surveys, systematic errors, most of the time evidenced through a tail queue Gaussian distribution. 
Since a SAM-based model contains more unknown parameters to estimate than the number of determined equations, updating and balancing such stochastically unbalanced matrices belongs to the category of the generalized inverse problem. 